\title{
Análise epidemiológica das internações hospitalares por doença isquêmica do coração no Piauí entre 2017 e 2020
}

\section{Epidemiological analysis of hospital admissions for ischemic heart disease in Piauí between 2017 and 2020}

Recebimento dos originais: 01/05/2021

Aceitação para publicação: 31/06/2021

\author{
Milena Viana Freire \\ Instituição: Centro Universitário Uninovafapi (UNINOVAFAPI), Teresina-Piauí. \\ Mariana de Sousa Meneses Carvalho \\ Instituição: Centro Universitário Uninovafapi (UNINOVAFAPI), Teresina-Piauí. \\ Aureliano Machado de Oliveira \\ Instituição: Centro Universitário Uninovafapi (UNINOVAFAPI), Teresina-Piauí.
}

\begin{abstract}
RESUMO
As doenças isquêmicas do coração (DIC) estão entre as principais causas de internações hospitalares no Piauí. Os índices são mais expressivos quanto maior a exposição aos fatores de risco cardiovasculares, o que ressalta a importância da adoção de hábitos mais saudáveis.
\end{abstract}

Palavras-chave: Doença isquêmica do coração, Internações, Epidemiologia.

\begin{abstract}
Ischemic heart diseases (IHD) are among the main causes of hospital admissions in Piauí. The higher the exposure to cardiovascular risk factors, the more expressive the indices are, which highlights the importance of adopting healthier habits.
\end{abstract}

Keywords: Ischemic Heart Disease, Hospitalizations, Epidemiology.

\section{INTRODUÇÃO}

As doenças cardiovasculares ocupam a quarta posição nas causas de internações hospitalares e constituem a principal causa de óbito no Brasil. Dentre elas, as doenças isquêmicas do coração estão entre as mais prevalentes (FIGUEIREDO FSF, et al., 2020).

As DIC caracterizam-se pela baixa oxigenação do músculo cardíaco devido ao desequilíbrio entre oferta e demanda de oxigênio. Sua principal etiologia é a aterosclerose nas artérias coronárias, que resulta na interrupção do fluxo sanguíneo e na consequente isquemia cardíaca (GONÇALVES PRT, et al., 2018). A formação da placa aterosclerótica envolve a interação de vários fatores de risco, que podem ser modificáveis, como sedentarismo, obesidade, hipertensão arterial sistêmica, diabetes mellitus, 
hiperlipidemia, tabagismo e estresse; e não modificáveis, que são os fatores hereditários, a idade e o sexo (SILVEIRA EL, et al., 2018).

A elevada incidência de DIC pode ser explicada pela crescente exposição aos fatores de risco relacionados com hábitos de vida. Outra razão é a mudança na estrutura etária da população, com aumento da proporção de idosos (SANTOS J, et al., 2018). Dessa forma, conhecer o perfil desses pacientes é fundamental para o planejamento de medidas de prevenção e de controle das doenças.

\section{OBJETIVO}

Analisar o perfil epidemiológico de pacientes internados por doença isquêmica do coração no Piauí, no período de janeiro de 2017 a dezembro de 2020, assim como as principais vertentes dessas internações hospitalares.

\section{MÉTODO}

Trata-se de um estudo epidemiológico do tipo retrospectivo e com abordagem quantitativa. Os dados foram coletados através de registros contidos no Sistema de Informações Hospitalares do SUS (SIH/SUS) entre 2017 e 2020, disponíveis no Departamento de Informática do SUS (DATASUS). As variáveis estudadas foram: número de internações, ano de atendimento, município, CID-10, faixa etária, sexo, número de óbitos, taxa de mortalidade e valor dos serviços hospitalares. Para a análise comparativa, os dados extraídos foram tabelados em números absolutos e percentuais por meio do software Microsoft Office Excel 2010. A submissão ao Comitê de Ética em Pesquisa (CEP) não foi necessária, uma vez que a base de dados utilizada no estudo é de domínio público.

\section{RESULTADOS}

Entre 2017 e 2020, foram registradas 11.623 internações por doenças isquêmicas do coração no Piauí. Dentre as quais o infarto agudo do miocárdio foi o mais frequente (63\%). E o município com maior percentual de internações foi Teresina $(81,57 \%)$.

No gráfico 1, pode-se observar a maior prevalência de internações no sexo masculino $(63,93 \%)$ em comparação com o sexo feminino (36,07\%). Também é possível notar que o número de internações aumenta com o avançar da idade. A faixa etária mais acometida, tanto para homens, quanto para mulheres, foi a de 60 a 69 anos.

No período analisado, registrou-se 621 óbitos, o que representa uma taxa de mortalidade de 5,34\%. Do total de óbitos, 80,83\% (502) são pacientes com mais de 60 anos.

Quanto às despesas hospitalares, o valor total referente aos quatro anos foi de R $25.174 .024,08$, com maior custo em 2019 ( $\mathrm{R} \$ 6.924 .813,80)$ e menor custo em 2020 ( $\mathrm{R} \$ 5.712 .134,98)$. 
Gráfico 1 - Internações hospitalares por doença isquêmica do coração no Piauí de acordo com sexo e faixa etária:

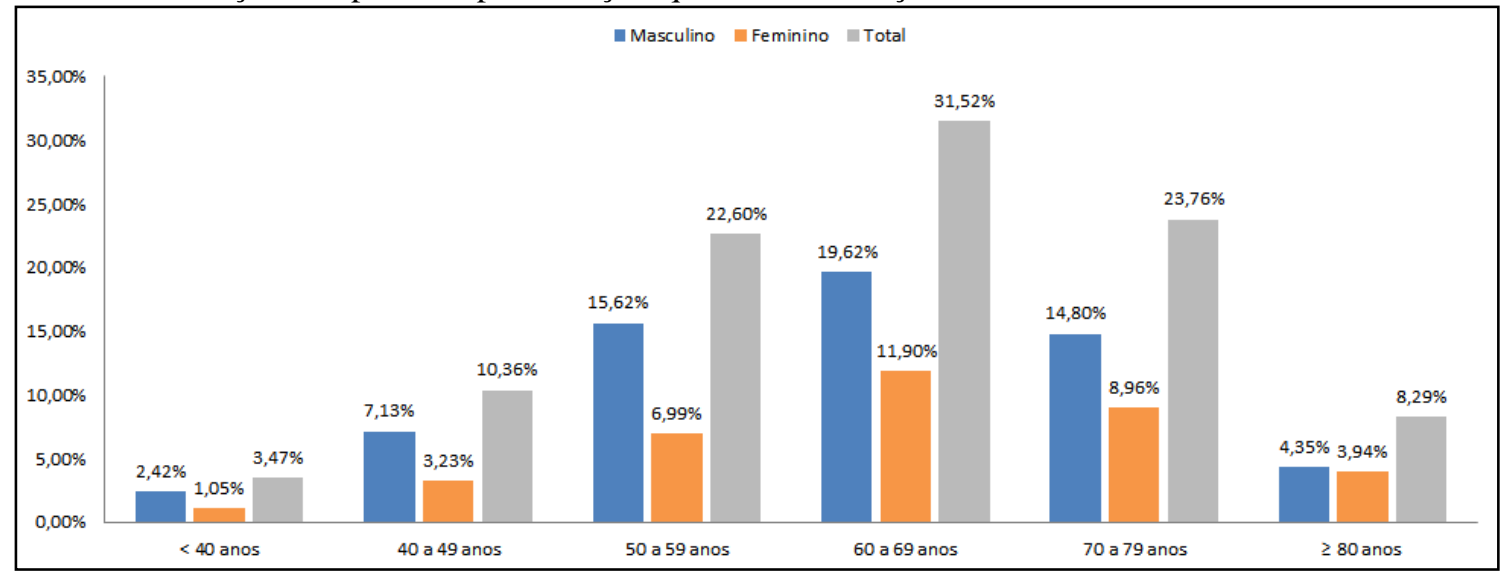

Fonte: FREIRE MV, et al., 2021. [Dados extraídos do DATASUS, 2021]

\section{DISCUSSÃO}

Teresina apresentou um percentual significativo de internações por DIC no Piauí. Esse resultado pode estar relacionado com a maior urbanização da capital, quando comparada com os outros municípios, o que favorece a adoção de hábitos de vida prejudiciais à saúde (SILVEIRA EL, et al.,2018).

A incidência foi maior nas faixas etárias mais avançadas. Isso porque a isquemia cardíaca está relacionada com o acúmulo de exposição aos fatores de risco no decorrer da vida (SANTOS J, et al., 2018).

Entre os sexos, o masculino foi o mais acometido. Devido a aspectos socioculturais, os homens geralmente apresentam maior resistência ao cuidado à saúde e são mais expostos a fatores como sobrepeso, sedentarismo e tabagismo, potencializando o risco de doenças cardiovasculares (MUSSI FC e TEXEIRA JRB, 2018).

As DIC representam importante impacto econômico à saúde pública. Internações decorrentes de doenças cardiovasculares são as que requerem maiores custos hospitalares (SIQUEIRA ASE, et al., 2017).

\section{CONSIDERAÇÕES FINAIS}

De acordo com o levantamento epidemiológico realizado, conclui-se que alguns grupos populacionais são mais vulneráveis ao acometimento por doenças isquêmicas do coração. Como é o caso de idosos, homens e moradores de zonas urbanas, visto que apresentam maior exposição aos fatores de risco cardiovasculares. Nesse panorama, faz-se necessário desenvolver estratégias preventivas e de controle da patologia - principalmente direcionando para esses grupos - de forma a reduzir os indicadores de incidência e mortalidade, bem como os custos por internações e outros serviços hospitalares. 


\section{REFERÊNCIAS}

1. FIGUEIREDO FSF, et al. Distribuição e autocorrelação espacial das internações por doenças cardiovasculares em adultos no Brasil. Revista Gaúcha de Enfermagem, 2020; 41: e20190314.

2. GONÇALVES PRT, et al. Aterosclerose e sua relação com as doenças cardiovasculares. Revista Saúde em Foco, 2018; 10: p. 711-717.

3. MUSSI FC, TEIXEIRA JRB. Fatores de risco cardiovascular, doenças isquêmicas do coração e masculinidade. Revista Cubana de Enfermería, 2018; 34 (2): 1-14.

4. SANTOS J, et al. Mortalidade por infarto agudo do miocárdio no Brasil e suas regiões geográficas: análise do efeito da idade-período-coorte. Ciência \& Saúde Coletiva, 2018; 23: p. 1621-1634.

5. SILVEIRA EL, et al. Prevalência e distribuição de fatores de risco cardiovascular em portadores de doença arterial coronariana no Norte do Brasil. Revista da Faculdade de Ciências Médicas de Sorocaba, 2018; 20(3): p. 167-173.

6. SIQUEIRA ASE, et al. Análise do impacto econômico das doenças cardiovasculares nos últimos cinco anos no Brasil. Arquivos Brasileiros de Cardiologia, 2017; 109 (1): p. 39-46. 